\title{
Some connections between classical and quantum anholonomy
}

\author{
G. Giavarini \\ Istituto Nazionale di Fisica Nucleare Gruppo Collegato di Parma, Dipartimento di Fisica Università di Parma, Parma, Italy \\ E. Gozzi \\ CERN, Geneva, Switzerland \\ D. Rohrlich* \\ Niels Bohr Institute, Copenhagen, Denmark \\ W. D. Thacker \\ Fachbereich Physik, Universität Kaiserslautern, Kaiserslautern, Federal Republic of Germany
}

(Received 22 August 1988)

\begin{abstract}
In this paper we study the interplay between the classical and quantum anholonomy effects (Hannay's angle and Berry's phase). When a quantum system with a finite number of energy levels has a Berry phase, it also has a nonzero Hannay angle. We show how systems with infinitely many levels can evade this correspondence, and find some necessary conditions for a system with a Berry phase to have no Hannay angle.
\end{abstract}

\section{INTRODUCTION}

Over the last four years there has been a great deal of interest in a new quantum effect known as Berry's phase. ${ }^{1}$ This phenomenon occurs in systems which depend on a number of external parameters $\left\{B_{l}\right\}$ and consists of an additional phase $\gamma_{n}$ acquired by the wave function of the system during adiabatic transport around a closed loop in parameter space.

In a subsequent development of this idea, Hannay ${ }^{2}$ and Berry $^{2}$ found a classical counterpart of the quantum effect. This classical phenomenon consists, for integrable systems, of an extra shift $\Delta \Theta_{j}$ picked up by the angle variables $\Theta_{j}$ as the parameters undergo a closed adiabatic excursion. These $\Delta \Theta_{j}$ are now called Hannay's angles. For a review and more details see Refs. 3 and 4.

Berry ${ }^{2}$ brought the analogy between these two effects a step further by showing that the semiclassical limit of Berry's phase $\gamma_{n}$ is related to Hannay's angle, the precise relationship being

$\frac{\partial \gamma_{\{n\}}}{\partial n_{j}}=\Delta \Theta_{j}+D(\{I\}, \mathcal{C}) \hbar^{2}+E(\{I\}, \mathcal{C}) \hbar^{4}+\cdots$,

where $C$ is the loop in parameter space while $\{n\}$ is the set of quantum numbers associated with the wave function $\psi_{\{n\}}$ and related to the classical action variables through the Bohr-Sommerfeld condition

$$
I_{j}=\left(n_{j}+\mu_{j}\right) \hbar,
$$

where the index $j$ labels the classical degrees of freedom.

Equation (1) is quite instructive because it tells us that if the classical effect is present $\left(\Delta \Theta_{j} \neq 0\right)$, then the quantum effect is also there. It tells us nothing about the converse question: If Berry's phase is different from zero, should we expect a nonzero Hannay angle? This is the kind of question we address in this paper.

In Sec. I we show that if one considers the dynamics generated by a time-varying Hamiltonian it is necessary to embed the starting Hamiltonian into a wider class, in order to identify the effective parameter space where the Berry-phase two-form has its singularities. Section II is devoted to the proof of some theorems concerning the dependence of the phase two-form on energy levels. We also show that a quantum system with a finite number of levels has a straightforward classical counterpart in terms of Grassmann variables and show explicitly how to evaluate Hannay's angle using group-theoretical techniques. In Sec. III we discuss the displaced harmonic oscillator as an example of the $N \rightarrow \infty$ limit of a system with $N$ levels and show how it evades the theorems pertaining to systems with a finite number of levels. In the Appendix we give the WKB expansion of Berry's phase in systems with one degree of freedom.

\section{THE ENLARGED PARAMETER SPACE}

In his seminal paper ${ }^{1}$ Berry pointed out a connection between quantum anholonomy and degeneracies in the parameter space. Berry's phase can be written as a surface integral

$$
\gamma_{n}=-\iint_{\rho^{\prime}} V_{n}
$$

where $\mathcal{S}$ is any surface in the parameter space bordered by the loop $\mathcal{C}$, and

$$
V_{n}=-i \sum_{\substack{m \\ n \neq m}} \frac{\langle n|d H| m\rangle \wedge\langle m|d H| n\rangle}{\left(E_{n}-E_{m}\right)^{2}}
$$


is called the phase two-form, $d$ being the exterior derivative in parameter space and $|n\rangle$ the instantaneous eigenstates of the parameter-dependent Hamiltonian. From (2) we see that $V_{n}$ develops singularities where levels cross, due to the vanishing energy denominator. Moreover, as Berry showed, the singularities act as monopole sources for the phase two-form.

On the other hand, one can imagine many cases where the parameter space contains no degeneracy and yet some degeneracy outside the parameter space acts as a source for the phase two-form. For example, consider the model

$$
H=B_{1} \sigma_{1}+B_{2} \sigma_{2}+\sigma_{3} .
$$

Here the parameter space is two dimensional and for no value of $\left(B_{1}, B_{2}\right)$ does the system develop a degeneracy. Nevertheless, a calculation of the phase two-form for the spin-up and spin-down states $| \pm\rangle$ yields

$$
V_{ \pm}=\mp \frac{d B_{1} \wedge d B_{2}}{\left(1+B_{1}^{2}+B_{2}^{2}\right)^{3 / 2}},
$$

which is the restriction to the $B_{3}=1$ plane of the monopole two-form arising from $H=B_{1} \sigma_{1}+B_{2} \sigma_{2}+B_{3} \sigma_{3}$. All the loops of (4) lie in the plane $B_{3}=1$, but still the monopole sitting at the origin makes itself felt in (5).

The same thing happens with the system described by the Hamiltonian ${ }^{5}$

$$
\begin{aligned}
H= & \frac{1}{2} \omega^{2} q^{2}[\cosh (2 \tau)-\sinh (2 \tau) \cos \theta] \\
& +\frac{1}{2} \omega(q p+p q) \sinh (2 \tau) \sin \theta \\
& +\frac{1}{2} p^{2}[\cosh (2 \tau)+\sinh (2 \tau) \cos \theta]
\end{aligned}
$$

The parameter space is a hyperboloid with coordinates $(\tau, \theta)$. The degeneracies are connected with the Hamiltonian

$$
H=\frac{1}{2}\left[X_{1} q^{2}+X_{2}(q p+p q)+X_{3} p^{2}\right]
$$

and they lie on the cone that is asymptotic to the hyperboloid sheets. This cone is outside the parameter space but nevertheless gives rise to a $V$ different from zero ${ }^{5}$ for the Hamiltonian (6).

It is possible for degeneracies lying outside the parameter space not to give rise to a phase two-form. This happens when the parameter space of the system is parallel to the flux generated by the degeneracies. An example of this is the model

$$
H=B_{1} \sigma_{1}+B_{2} \sigma_{2} \quad\left(B_{1}>0\right) .
$$

The flux $\mathbf{V}$ generated by the monopole at the origin is always parallel to the half-plane in which the model lives.

From both examples (4) and (6), we see that the phase two-form felt by the system originates from degeneracies of an enlarged Hamiltonian living in an enlarged parameter space. (We thank E. Kiritsis for suggesting this idea long ago in a private correspondence.) What determines the effective parameter space and Hamiltonian of a system?

To answer this question, ${ }^{6}$ consider the exact evolution operator for a time-dependent Hamiltonian,

$$
U(T)=\mathcal{T} \exp \left[-i \int_{0}^{T} H(t) d t\right],
$$

where $\mathcal{T} \exp$ denotes the time-ordered exponential. It can be computed using the Magnus formula ${ }^{7}$

$$
\begin{aligned}
U(T)=\exp [ & -i \int_{0}^{T} H(t) d t \\
& \left.-\frac{1}{2} \int_{0}^{T} \int_{0}^{t}\left[H(t), H\left(t^{\prime}\right)\right] d t d t^{\prime}+\cdots\right],
\end{aligned}
$$

where the higher-order terms contain nested commutators such as $\left[H(t),\left[H\left(t^{\prime}\right), H\left(t^{\prime \prime}\right)\right]\right]$. So we see that new terms come into the evolution operator from the multiple commutators of $H$ with itself at different times.

Doing this for the Hamiltonian (4), we see that $U(T)$ contains terms such as

$$
\begin{aligned}
{\left[H(\mathbf{B}), H\left(\mathbf{B}^{\prime}\right)\right]=} & \sigma_{1}\left(-B_{2}^{\prime}+B_{2}\right)+\sigma_{2}\left(B_{1}^{\prime}-B_{1}\right) \\
& +\sigma_{3}\left(B_{1} B_{2}^{\prime}-B_{1}^{\prime} B_{2}\right) .
\end{aligned}
$$

Thus the commutator has effectively generated a parameter multiplying $\sigma_{3}$, which was not present in the original Hamiltonian (4). The enlarged parameter space felt by the system is $\left\{B_{1}, B_{2}, B_{3}\right\}$ and contains a degeneracy of the effective Hamiltonian

$$
H=B_{1} \sigma_{1}+B_{2} \sigma_{2}+B_{3} \sigma_{3},
$$

which acts as a source for the two-form (5).

Therefore, we can prescribe a rule for generating the enlarged parameter space and Hamiltonian. We take nested commutators between terms of the original Hamiltonian until the Lie algebra closes, and then assign a parameter to each generator of the algebra. In general the starting Hamiltonian can be written as

$$
H=\sum_{i=1}^{g} B_{i} T^{i},
$$

where $T^{i}$ are generators of a Lie algebra of dimension $n$. Then the effective Hamiltonian felt by the system is

$$
H^{\prime \prime}=\sum_{i=1}^{g} B_{i} T^{i}+\sum_{j=g+1}^{n} B_{j} T^{j} .
$$

This means we have to add the generators $T^{j}$ multiplied by the new parameters $B_{j}(j=g+1, \ldots, n)$ which close the algebra and make all the parameters independent.

For the Hamiltonian (4) the algebra is su(2), for (6) it is $\mathrm{su}(1,1)$ and, for the displaced harmonic oscillator, ${ }^{5}$

$$
H=\frac{1}{2}\left\{\left[p-(\hbar \omega)^{1 / 2} X_{2}\right]^{2}+\omega^{2}\left[q-(2 \hbar / \omega)^{1 / 2} X_{1}\right]^{2}\right\}
$$

it is e(2), the algebra of the Euclidean group in two dimensions. Of course, it can happen that the algebra does not close and so the effective Hamiltonian contains an infinite number of parameters. An example of this is

$$
H=\alpha p^{2}+\beta(p q+q p)+\gamma q^{2}+\delta q^{4} .
$$

To summarize, as it should already be clear from the examples given above, the reason for enlarging the parameter space is to look for degeneracies lying outside the 
original space which act as sources for the phase twoform. Our procedure ensures that all degeneracies will be contained within the enlarged parameter space.

\section{THEOREMS}

Using this definition of the effective parameter space we study the relationship between Berry's phase and Hannay's angle. We shall restrict our considerations to complex Hamiltonians. In fact, when a pseudoreal Hamiltonian is extended according to the procedure of Sec. I it becomes complex.

We first note that Hannay's angle is not directly related to Berry's phase, but to its derivative with respect to $n$. This means that the classical effect is only there when $\gamma$ depends on $n$. Berry's phase does not always depend on $n$, in fact in the displaced harmonic oscillator ${ }^{5}$ it is nonzero and the same for all levels. Thus we are led to study the dependence of Berry's phase on energy levels.

Within this context we formulate the following.

Theorem 1: If a system has a finite number of levels and the phase two-form $V_{n}$ is independent of $n$, then $V_{n}=0$.

Proof: We write the phase two-form $V_{n}$ as

$$
V_{n}=\sum_{k \neq n} V_{n k}
$$

with

$$
V_{n k}=\frac{-i\langle n|d H| k\rangle \wedge\langle k|d H| n\rangle}{\left(E_{n}-E_{k}\right)^{2}},
$$

and note that $V_{n k}=-V_{k n}$. If we suppose that $V_{n}$ is independent of $n$, then

$$
V_{n}=V=\sum_{k \neq n} V_{n k}
$$

Now summing over $n$ and supposing that the total number of levels is $N$, we get

$$
\sum_{n=1}^{N} V_{n}=N V=\sum_{n, k=1}^{N} V_{n k}=0
$$

from the antisymmetry of $V_{n k}$, and therefore $V=0$.

The condition that the number of levels be finite is essential because otherwise (10) is the sum of an infinite series and we cannot conclude that it gives zero. In Sec. III we shall show explicitly how the displaced harmonic oscillator, which has an infinite number of levels, gets around this theorem.

Theorem 1 tells us that for systems with a finite number of levels Berry's phase can only be nonzero if it depends on $n$. From this we obtain the following.

Theorem 2: If a system has a finite number of levels and if Berry's phase is nonzero, then Hannay's angle is also nonzero.

Proof: Theorem 1 implies that $\partial \gamma_{n} / \partial n \neq 0$. This does not yet mean that Hannay's angle exists since, as we have already mentioned,

$$
\frac{\partial \gamma_{n}}{\partial n}=\Delta \Theta+\text { higher-order terms in } \hbar,
$$

and it might be that $\Delta \theta=0$ while some of the higherorder terms are nonzero.

So we find it necessary to show explicitly how to construct Hannay's angle for the classical version of a system with a finite number of levels. We follow the method of the second paper in Ref. 4. For a general $N$-level system the quantum Hamiltonian is an element of the algebra $\mathrm{su}(N)$ plus a multiple of the identity. ${ }^{8}$ The multiple of the identity is a (time-dependent) vacuum energy which does not affect Berry's phase, as can be seen by noting that in expression (3) for $V_{n}$ only off-diagonal matrix elements of $d H$ appear and therefore, a multiple of the identity in $H$ will not contribute to the phase two-form. Thus we can renormalize the quantum Hamiltonian by subtracting the vacuum energy and write it in the form

$$
\hat{H}=\hbar B^{a} T^{a},
$$

where $T^{a}$ are generators of $\operatorname{su}(N)$ in one of the $N$ dimensional fundamental representations, satisfying

$$
\left[T^{a}, T^{b}\right]=i f^{a b c} T^{c},
$$

$f^{a b c}$ being the totally antisymmetric structure constants of $\operatorname{su}(N)$.

In order to find Berry's phase for the system (11) we diagonalize the Hamiltonian

$$
\hat{H}_{\text {diag }}(B)=\hat{U}^{\dagger}(B) \hat{H}(B) \hat{U}(B),
$$

where $\hat{H}_{\text {diag }}$ contains the energy levels

$$
\mathscr{E}_{k}=\widehat{U}_{k i} \hat{H}_{i j} \hat{U}_{j k}, i, j, k=1, \ldots, N(\text { no sum on } k) .
$$

$\hat{U}$ is a unitary $N \times N$ matrix whose columns are the eigenvectors $|k\rangle$ of the matrix $\hat{U}(B)$ :

$$
\hat{H}|k\rangle=\mathscr{E}_{k}|k\rangle \text { or } \hat{H}_{i j} \hat{U}_{j k}=\mathscr{E}_{k} \hat{U}_{i k} \text {. }
$$

The Berry phase one-form is then given by

$$
A_{k}=-i\langle k|d| k\rangle=-i \sum_{j=1}^{N} \hat{U}_{k j}^{\dagger} d \hat{U}_{j k}
$$

and Berry's phase for the circuit $\mathcal{C}$ can be written as the line integral

$$
\gamma_{k}(\mathcal{C})=-\oint_{e} A_{k} \text {. }
$$

To construct a classical version of (11) we introduce $N^{2}-1$ real Grassmann variables ${ }^{9} \psi^{a}$ which satisfy

$$
\left\{\psi^{a}, \psi^{b}\right\}=\psi^{a} \psi^{b}+\psi^{b} \psi^{a}=0
$$

and the Poisson brackets

$$
\left\{\psi^{a}, \psi^{b}\right\}_{\mathrm{PB}}=i \delta^{a b} .
$$

We build up from these Grassmann variables the classical Lie-algebra generators

$$
\mathcal{T}^{a}=-\frac{i}{2} f^{b c a} \psi^{b} \psi^{c},
$$

which satisfy

$$
\left\{\mathcal{T}^{a}, \mathcal{T}^{b}\right\}_{\mathrm{PB}}=-f^{a b c} \mathcal{T}^{c} .
$$


When the theory is quantized these classical generators go over into the quantum generators $\hbar T^{a}$ and $\left\{\mathcal{T}^{a}, \mathcal{T}^{b}\right\}_{\mathrm{PB}} \rightarrow(i / \hbar)\left[\hbar T^{a}, \hbar T^{b}\right]$.

The classical version of (11) is then

$$
H=B^{c} \mathcal{T}^{c}=-\frac{i}{2} \psi^{a} \psi^{b} f^{a b c} B^{c}=\frac{1}{2} \psi^{a} \psi^{b} H_{a b}
$$

corresponding to the classical Lagrangian

$$
L=\frac{i}{2} \psi^{a} \dot{\psi}^{a}+\frac{i}{2} \psi^{a} \psi^{b} f^{a b c} B^{c},
$$

from which we derive the canonical momenta, $\pi^{a}=\mathrm{\partial} L / \partial \dot{\psi}^{a}=(i / 2) \psi^{a}$ (right derivative).

To derive Hannay's angle for this model we perform at each point in parameter space a canonical transformation to normal modes

$$
\widetilde{\psi}_{\alpha}=U_{\alpha a}^{\dagger} \psi_{a}, \quad \widetilde{\psi}_{\alpha}^{*}=\psi_{a} U_{a \alpha}
$$

where $U(B)$ is a unitary $\left(N^{2}-1\right) \times\left(N^{2}-1\right)$ matrix which diagonalizes $H(B)$ :

$$
\lambda_{\alpha}=U_{\alpha a}^{\dagger} H_{a b} U_{b \alpha}
$$

(no sum on $\alpha$ ). In terms of normal modes the instantaneous Hamiltonian and Lagrangian become

$$
\begin{aligned}
& \mathcal{H}=\frac{1}{2} \lambda_{\alpha} \widetilde{\psi}_{\alpha}^{*} \widetilde{\psi}_{\alpha}, \\
& \mathcal{L}=\frac{i}{2} \widetilde{\psi}_{\alpha}^{*} \dot{\bar{\psi}}_{\alpha}-\frac{1}{2} \lambda_{\alpha} \widetilde{\psi}_{\alpha}^{*} \widetilde{\psi}_{\alpha}
\end{aligned}
$$

yielding the equations of motion

$$
\dot{\bar{\psi}}_{\alpha}=-i \lambda_{\alpha} \widetilde{\psi}_{\alpha}, \quad \dot{\bar{\psi}}_{\alpha}^{*}=i \lambda_{\alpha} \widetilde{\psi}_{\alpha}^{*}
$$

with the solutions

$$
\widetilde{\psi}_{\alpha}(t)=e^{-i \lambda \alpha^{t}} \widetilde{\psi}_{\alpha}(0), \quad \widetilde{\psi}_{\alpha}^{*}(t)=e^{i \lambda \alpha^{t}} \widetilde{\psi}_{\alpha}^{*}(0)
$$

The normal modes have canonical momenta $\widetilde{\pi}_{\alpha}$ $=\partial \mathcal{L} / \partial \dot{\widetilde{\psi}}_{\alpha}=(i / 2) \widetilde{\psi}_{\alpha}^{*}$ (right derivative) and actions

$$
I_{\alpha}=\frac{1}{2 \pi} \oint_{d t} \widetilde{\pi}_{\alpha}(t) \dot{\vec{\psi}}_{\alpha}(t)=\frac{1}{2} \widetilde{\psi}_{\alpha}^{*} \widetilde{\psi}_{\alpha},
$$

in terms of which the instantaneous Hamiltonian is given by $\mathscr{H}=\lambda_{\alpha} I_{\alpha}$. The angle variables obey the instantaneous equation of motion $\dot{\Theta}_{\alpha}=\partial \mathcal{H} / \partial I_{\alpha}=\lambda_{\alpha}$ which has the solution $\Theta_{\alpha}(t)=\lambda_{\alpha} t$. The normal modes are given in terms of the angle variables by

$$
\widetilde{\psi}_{\alpha}(\Theta)=e^{-i \Theta_{\alpha}} \widetilde{\psi}_{\alpha}(0), \quad \widetilde{\psi}_{\alpha}^{*}(\Theta)=e^{i \Theta_{\alpha}} \widetilde{\psi}_{\alpha}^{*}(0)
$$

When the parameters are then changed adiabatically around a closed loop $\mathcal{C}$ the angle variables $\Theta_{\alpha}$ pick up an extra shift (Hannay's angle) given by

$$
\begin{aligned}
& \Delta \Theta_{\alpha}(\mathcal{C})=\oint_{\odot} \mathcal{A}_{\alpha}, \\
& \text { where }^{2,4}\left(M=N^{2}-1\right) \\
& \mathcal{A}_{\alpha}=-\frac{\partial}{\partial I_{\alpha}} \oint \frac{d \Theta_{1} \cdots d \Theta_{M}}{(2 \pi)^{M}} \sum_{b=1}^{N} \pi^{b}(\Theta, I ; B) d_{B} \psi^{b}(\Theta, I ; B) \\
& =-i \sum_{a=1}^{N} U_{\alpha a}^{\dagger} d U_{a \alpha}
\end{aligned}
$$

is the angle one-form.

Let us now take a closer look at the matrix $U$ which diagonalizes the classical Hamiltonian. It satisfies

$$
-i f^{a b c} B^{c} U_{b \alpha}=\lambda_{\alpha} U_{a \alpha}
$$

(no sum on $a$ and $\alpha$ ), which is the secular equation ${ }^{10}$ for the roots of the algebra $\operatorname{su}(N)$. We need to relate $U$ with the eigenstates of the quantum Hamiltonian (11). $N-1$ of the eigenvalues $\lambda_{\alpha}$ are zero, corresponding to the $N-1$ elements of the Cartan subalgebra. The remaining $N^{2}-1-(N-1)=N(N-1)$ eigenvalues split into positive and negative pairs called the roots of the Lie algebra. For $\lambda_{\alpha}=0, T^{a} U_{a \alpha}$ (summed over $a$ ) is the corresponding element of the Cartan subalgebra while for $\lambda_{\alpha} \neq 0, T^{a} U_{a \alpha}$ is the corresponding ladder operator, as can be seen from the following consideration:

$$
\begin{aligned}
\hat{H} T^{a} U_{a \alpha}|k\rangle & =T^{a} U_{a \alpha} \hat{H}|k\rangle+\left[\hat{H}, T^{a} U_{a \alpha}\right]|k\rangle \\
& =\left(\mathscr{E}_{k}+\lambda_{\alpha}\right) T^{a} U_{a \alpha}|k\rangle,
\end{aligned}
$$

with $\hat{H}$ defined in (11). Therefore,

$$
\left(T^{a}\right)_{i j} U_{a \alpha} \widehat{U}_{j k}=\eta_{\alpha, k} \widehat{U}_{i l},
$$

where $l$ and $k$ are related by $\mathscr{E}_{l}-\mathscr{E}_{k}=\lambda_{\alpha}$ and

$$
\eta_{\alpha, k}=\left[\hat{U}_{k i}\left(T^{b}\right)_{i j} U_{\alpha b}^{\dagger}\left(T^{a}\right)_{j m} U_{a \alpha} \hat{U}_{m k}\right]^{1 / 2}
$$

is a normalization constant, independent of $B$.

The $N(N-1)$ roots (and hence, the nonzero Hannay angles) are not all independent but can be expressed in terms of $N-1$ simple roots (and Hannay angles). We choose these simple roots to correspond to raising operators between an energy level and the next higher level. Suppose that $\lambda_{\alpha}=\mathscr{E}_{k+1}-\mathscr{E}_{k}$ is one such root. Then a straightforward calculation yields

$$
\begin{aligned}
A_{k+1}-A_{k}= & -i\left(\hat{U}_{k+1 j}^{\dagger} d \hat{U}_{j k+1}-\hat{U}_{k j}^{\dagger} d \hat{U}_{j k}\right) \\
= & -i\left\{\eta_{\alpha, k}^{-2}\left[\hat{U}_{k i}^{\dagger} U_{\alpha a}^{\dagger}\left(T^{a}\right)_{i j}\left(T^{b}\right)_{j m} U_{b \alpha} d \hat{U}_{m k}+\hat{U}_{k i}^{\dagger} U_{\alpha a}^{\dagger}\left(T^{a}\right)_{i j}\left(T^{b}\right)_{j m} \hat{U}_{m k} d U_{b \alpha}\right]-\hat{U}_{k j}^{\dagger} d \hat{U}_{j k}\right\} \\
=-i\left\{\eta _ { \alpha , k } ^ { - 2 } \left[\hat{U}_{k i}^{\dagger} U_{\alpha a}^{\dagger}\left(T^{a}\right)_{i j}\left(T^{b}\right)_{j m} U_{b \alpha} \hat{U}_{m l} \hat{U}_{l n}^{\dagger} d \hat{U}_{n k}\right.\right. & \\
& \left.\left.\quad+\hat{U}_{k i}^{\dagger} U_{\alpha a}^{\dagger}\left(T^{a}\right)_{i j}\left(T^{c}\right)_{j m} U_{c \beta} \hat{U}_{m k} U_{\beta b}^{\dagger} d U_{b \alpha}\right]-\hat{U}_{k j}^{\dagger} d \hat{U}_{j k}\right\}=-i U_{\alpha a}^{\dagger} d U_{a \alpha}=\mathcal{A}_{\alpha}
\end{aligned}
$$


(no sum on $k$ and $\alpha$ ). Therefore, we have found that the $N$-level system with quantum Hamiltonian (11) and classical Hamiltonian (13) satisfies the exact semiclassical relation

$$
\gamma_{k+1}(\mathcal{C})-\gamma_{k}(\mathcal{C})=-\Delta \Theta_{\alpha}(\mathcal{C}), \quad \lambda_{\alpha}=\mathscr{E}_{k+1}-\mathscr{E}_{k}
$$

[where the minus sign comes from our convention $\left.\widetilde{\psi}_{\alpha}(\Theta)=e^{-i \Theta_{\alpha}} \widetilde{\psi}(0)\right]$.

In the next theorem we relax the condition that the system have a finite total number of levels.

Theorem 3: If accidental degeneracies involving a finite number of levels are the sources for the phase twoform $V_{n}$, then $V_{n}$ and $\gamma_{n}$ will depend on $n$.

Proof: Suppose that at some point $B^{*}$ in the effective parameter space the $K$ levels $|n\rangle(n=1, \ldots, K)$ become degenerate. Then in the neighborhood of $B^{*}$ the dominant terms in $V_{n}$ will contain the small energy denominators $\left(E_{n}-E_{m}\right)^{2}(n, m=1, \ldots, K)$. Therefore,

$$
V_{n}\left(B \approx B^{*}\right)=\sum_{m \neq n}^{K} V_{n m}
$$

up to small terms. Assume that the phase two-forms for all the near-degenerate levels are the same. Then summing them we get, as in the proof of Theorem 1,

$$
\sum_{n=1}^{K} V_{n}=K V=0
$$

and hence $V=0$. This contradicts the assumption that the degeneracy is a source for $V_{n}$. Therefore, the phase two-forms of the near-degenerate levels cannot all be the same and $V_{n}$ has a dependence on $n$.

In this theorem it is necessary that the number of levels involved in the degeneracy be finite, otherwise we cannot conclude that the sum of the phase two-forms of the levels involved is zero.

Theorem 3 tells us that if $V_{n}$ does not depend on $n$ it cannot have its source in a degeneracy involving a finite number of levels. This statement also applies to the displaced harmonic oscillator where, as we shall see in the next section, the source of $V_{n}$ comes from a degeneracy involving an infinite number of levels.

\section{THE DISPLACED HARMONIC OSCILLATOR}

In this section we study the displaced harmonic oscillator as an interesting example of how the theorems of the previous section can be evaded in a system with an infinite number of levels. The model is ${ }^{5}$

$$
H=\hbar \omega\left[\left(a^{\dagger}-\alpha^{*}\right)(a-\alpha)+\frac{1}{2}\right],
$$

where $\alpha=X_{1}+i X_{2}$, and $X_{1}, X_{2}$ are slowly varying parameters. Chaturvedi, Sriram, and Srinivasan ${ }^{5}$ calculate the Berry phase to be

$$
\begin{aligned}
\gamma_{n}(\mathcal{C}) & =2 \int_{\mathcal{S}:(\mathrm{e}=e)} \int d X_{1} \wedge d X_{2} \\
& =2(\text { area of loop })
\end{aligned}
$$

independent of the energy level $n$. Therefore, Hannay's angle is zero.
Here there is apparently no degeneracy and we have to enlarge the parameter space according to the rule of Sec. I and Ref. 6. The algebra is a central extension of e(2), consisting of $\left\{a, a^{\dagger}, a^{\dagger} a, 1\right\}$. Assigning an independent parameter to each of these terms we have

$$
H=\hbar \omega a^{\dagger} a-\hbar \omega \alpha^{*} a-\hbar \omega \alpha a^{\dagger}+K .
$$

The parameter space is now $\left(\omega, X_{1}, X_{2}, K\right)$ and the degeneracy is at $\omega=0$. This degeneracy involves all of the infinite number of levels. So the system need not obey Theorem 3 about degeneracies involving a finite number of levels and, indeed, Berry's phase has no dependence on $n$.

In order to gain some more insight into this system, let us view it as the Lie-algebra contraction of the su(2) Hamiltonian $^{5,11}$

$$
H=\hbar \mathbf{J} \cdot \mathbf{B}=\hbar\left(B_{z} J_{z}+B_{-} J_{+}+B_{+} J_{-}\right)
$$

with

$$
J_{ \pm}=\frac{1}{\sqrt{2}}\left(J_{x} \pm i J_{y}\right), \quad B_{ \pm}=\frac{1}{\sqrt{2}}\left(B_{x} \pm i B_{y}\right)
$$

which has matrix elements between the states $|j m\rangle$ and $|j n\rangle$

$$
\begin{aligned}
& H_{n m}= \hbar B_{z} n \delta_{n m} \\
&+\frac{\hbar}{\sqrt{2}}\left\{B_{-}[j(j+1)-m(m+1)]^{1 / 2} \delta_{n, m+1}\right. \\
&\left.\quad+B_{+}[j(j+1)-m(m-1)]^{1 / 2} \delta_{n, m-1}\right\} .
\end{aligned}
$$

$H_{n m}$ is a finite-dimensional matrix, so we can relabel the states

$$
\begin{aligned}
n \rightarrow n^{\prime} & =n+j, \\
m \rightarrow m^{\prime} & =m+j, \\
H_{n^{\prime} m^{\prime}}= & \left(n^{\prime}-j\right) \hbar B_{z} \delta_{n^{\prime}, m^{\prime}} \\
& +\hbar \sqrt{j}\left[B_{-.}\left(m^{\prime}+1\right)^{1 / 2} \delta_{n^{\prime}, m^{\prime}+1}\right. \\
& \left.\quad+B_{+} \sqrt{m^{\prime}} \delta_{n^{\prime}, m^{\prime}-1}\right]+O(\hbar / \sqrt{j}) .
\end{aligned}
$$

Now we take the limit as $j \rightarrow \infty$ in such a way that $\sqrt{j} B_{ \pm} / B \rightarrow$ const. We drop the term $j B_{z} \delta_{n^{\prime}, m^{\prime}}$, an infinite renormalization of the energy. In this limit the matrix element $n^{\prime} \delta_{n^{\prime}, m^{\prime}}$ becomes $a^{\dagger} a$ while $\left(m^{\prime}+1\right)^{1 / 2} \delta_{n^{\prime}, m^{\prime}+1}$ and $\sqrt{m^{\prime}} \delta_{n^{\prime}, m^{\prime}-1}$ become, respectively, $a^{\dagger}$ and $a$. Thus we reproduce the displaced harmonic oscillator (up to a constant)

$$
H=\hbar \omega\left(a^{\dagger} a+\alpha a^{\dagger}+\alpha^{*} a\right),
$$

with

$$
\omega=B_{z} \approx B, \quad \alpha=\lim _{j \rightarrow \infty} \sqrt{j} \frac{B_{-}}{B}
$$

appearing as the $j \rightarrow \infty$ limit of $\hbar \mathbf{J} \cdot \mathbf{B}$.

For (17) the element $V_{n m}$ of the phase two-form is (in the neighborhood of $B_{x} / B=B_{y} / B=0$ ) 


$$
\begin{aligned}
V_{n m} & \left.=\operatorname{Im} \mid \frac{2}{B^{2}(m-n)^{2}}\left\langle n\left|\frac{\partial H}{\partial B_{x}}\right| m\right\rangle\left\langle m\left|\frac{\partial H}{\partial B_{y}}\right| n\right\rangle\right] d B_{x} \wedge d B_{y} \\
& =\frac{1}{2 B^{2}}\left\{[j(j+1)-m(m+1)] \delta_{n, m+1}+[j(j+1)-m(m-1)] \delta_{n, m-1}\right\} d B_{x} \wedge d B_{y} \\
& =\frac{j}{B^{2}}\left[\left(m^{\prime}+1\right) \delta_{n^{\prime}, m^{\prime}+1}-m^{\prime} \delta_{n^{\prime}, m^{\prime}-1}+O(n / j)\right] d B_{x} \wedge d B_{y} .
\end{aligned}
$$

The dependence on $n$ is now suppressed by a factor of $j$ and in this limit we get

$$
V_{n^{\prime}}=V_{n^{\prime}, n^{\prime}+1}+V_{n^{\prime}, n^{\prime}-1}=-\frac{j}{B^{2}} d B_{x} \wedge d B_{y} ;
$$

it does not vanish, yet it does not depend on $n^{\prime}$ and it agrees with (16).

At this point we should warn the reader that the topologies in the two cases considered $[\mathrm{E}(2)$ and $\mathrm{SU}(2)]$ are different. What we have done is a standard group contraction $^{11}$ which, in general, leads from one group to another one which is not isomorphic to it. In any case, since this calculation uses the local phase two-form, there are no global topological subtleties that might spoil the picture in going from $\mathrm{SU}(2)$ to $\mathrm{E}(2)$.

To summarize, we have related the displaced harmonic-oscillator Berry phase to a degeneracy. The degeneracy is the one causing all levels to collapse, i.e., $\omega=0$, because, relative to the $\mathbf{J} \cdot \mathbf{B}$ problem, this means $B_{z}=B_{x}=B_{y}=0$. But, since $B_{z} \approx B$ is scaled up from $B_{x}$ and $B_{y}$ by a factor of $\sqrt{j}$, this degeneracy is infinitely far away in the limit.

\section{CONCLUSION}

To understand the relationship between classical and quantum anholonomy, we have studied quantum systems with a finite number of energy levels. For these systems the correlation between Hannay's angle and Berry's phase is exact: if one is nonzero so is the other. But there are examples of quantum systems with an infinite number of levels which break this correspondence and the remaining questions have to do with the special propties of these systems. If the anholonomy arises because a finite number of levels becomes degenerate somewhere in parameter space, the Hamiltonian can be treated as finite dimensional in the neighborhood of the degeneracy. Thus only degeneracies among infinitely many levels concern us. The displaced harmonic oscillator is an explicit example of how such a system breaks the link between classical and quantum anholonomy and shows an infinite degeneracy. It would be very useful to understand the dependence of Berry's phase on energy level $n$ in the higher orders of $\hbar$, since Hannay's angle depends only on the semiclassical term. To go beyond the semiclassical limit we need a complete $\hbar$ expansion of the Berry phase. Such an expansion has not been published before, and we provide one in the Appendix. This expansion and the theorems presented above are a step towards understanding the special relationship between classical and quan- tum anholonomy for systems with infinitely many energy levels.

\section{ACKNOWLEDGMENTS}

E.G. would like to thank members of Institut de Physique Nucléaire in Orsay, where this work was begun, for their hospitality and especially A. Comtet for valuable discussions. G.G. thanks members of the Theory Division at CERN for their kind hospitality.

\section{APPENDIX: WKB EXPANSION OF BERRY'S PHASE}

In this appendix we consider the WKB expansion of Berry's phase one-form

$$
A=\operatorname{Im}(\langle n ; B|d| n ; B\rangle)
$$

for which the integral around a closed loop yields Berry's phase. The phase one-form is related to the phase twoform $V$ by $d A=V$.

For simplicity we restrict our considerations to systems with one degree of freedom and Hamiltonians of the form

$$
\begin{aligned}
H(p, q ; B) & =\frac{B_{1}}{2} p^{2}+\frac{1}{2}[f(q ; B) p+p f(q ; B)]+V(q ; B) \\
& =\frac{B_{1}}{2} p^{2}+f(q ; B) p+V(q ; B)+\frac{\epsilon}{2} f^{\prime}(q ; B),
\end{aligned}
$$

where $\epsilon=-i \hbar, B=\left\{B_{K}\right\}$ is a set of external parameters, the linear term in $p$ is there to ensure that the wave function be complex and Berry's phase exists, and the prime means derivative with respect to $q$.

It is convenient to use the WKB expansion of the wave function in the form given by Maslov and Fedoriuk: ${ }^{12}$

$$
\psi(q)=e^{S / \epsilon} \sum_{n=0}^{\infty} \epsilon^{n} \phi_{n}(q) .
$$

Substituting (A1) into the Schrödinger equation

$$
\begin{aligned}
& \frac{B_{1}}{2} \epsilon^{2} \psi^{\prime \prime}(q)+\epsilon f(q, B) \psi^{\prime}(q) \\
& +\left(V(q ; B)+\frac{\epsilon}{2} f^{\prime}(q ; B)-E\right) \psi(q)=0
\end{aligned}
$$

we get 


$$
\begin{aligned}
& \sum_{n=0}^{\infty} \epsilon^{n}\left\{\left[\frac{B_{1}}{2} S^{\prime 2}\right.\right.\left.+f S^{\prime}+V-E\right] \phi_{n} \\
&+\epsilon\left[\left[\frac{B_{1}}{2} S^{\prime \prime}+\frac{f^{\prime}}{2}\right] \phi_{n}+\left(B_{1} S^{\prime}+f\right) \phi_{n}^{\prime}\right] \\
&\left.+\frac{\epsilon^{2} B_{1}}{2} \phi_{n}^{\prime \prime}\right\}=0
\end{aligned}
$$

The $O\left(\epsilon^{0}\right)$ term in (A2) gives the Hamilton-Jacobi equation for $S$,

$$
\frac{B_{1}}{2} S^{\prime 2}+f S^{\prime}+V-E=0
$$

which has the solution

$$
S(q, I ; B)=\int_{q_{0}}^{q} d q^{\prime} p\left(q^{\prime}, I ; B\right),
$$

where

$$
\begin{aligned}
p(q, I ; B)= & -\frac{f(q ; B)}{B_{1}} \\
& \pm\left(\frac{f(q ; B)^{2}}{B_{1}^{2}}+\frac{2[E(I)-V(q ; B)]}{B_{1}}\right)^{1 / 2}
\end{aligned}
$$

and (A4) is understood to be a sum of integrals along different branches of the torus of action

$$
\begin{aligned}
I(E ; B) & =\int p(q, E ; B) d q \\
= & \int\left[-\frac{f(q ; B)}{B_{1}}\right. \\
& \left. \pm\left(\frac{f(q ; B)}{B_{1}^{2}}+\frac{2[E-V(q ; B)]}{B_{1}}\right]^{1 / 2}\right] d q
\end{aligned}
$$

(see Berry ${ }^{13}$ ). Equation (A6) can in principle be inverted to give $E(I)$ in (A5).

The coefficient of $\epsilon$ in (A2) gives

$$
\left[\frac{B_{1}}{2} S^{\prime \prime}+\frac{f^{\prime}}{2}\right] \phi_{0}+\left(B_{1} S^{\prime}+f\right) \phi_{0}^{\prime}=0
$$

or

$$
\frac{\phi_{0}^{\prime}}{\phi_{0}}=-\frac{1}{2} \frac{\pi^{\prime}}{\pi},
$$

where we have introduced

$$
\pi(q, I ; B)=p(q, I ; B)+\frac{f(q ; B)}{B_{1}},
$$

which is like a kinetic momentum with the "gauge field" $f(q ; B) / B_{1}$. The solution of (A7) has the form

$$
\phi_{0}(q, I ; B)=c[\pi(q, I ; B)]^{-1 / 2},
$$

which is proportional to the square root of the Jacobian for the transformation from configuration space to angle variables, as one can see by substituting (A5) and (A6) into

$$
\begin{aligned}
\frac{\partial \Theta(q, I ; B)}{\partial q}=\frac{\partial^{2} S(q, I ; B)}{\partial q \partial I} & =\frac{\partial p(q, I ; B)}{\partial I} \\
& =\frac{\partial p(q, E ; B) / \partial E}{\partial I(E ; B) / \partial E}
\end{aligned}
$$

We leave open the renormalization of the wave function and fix the constant $c$ so that

$$
\phi_{0}=\left(\frac{1}{2 \pi} \frac{\partial \Theta}{\partial q}\right)^{1 / 2}
$$

For $n \geq 1$ we obtain, from (A2),

$$
\phi_{n}^{\prime}+\frac{1}{2} \frac{\pi^{\prime}(q)}{\pi(q)} \phi_{n}=-\frac{\phi_{n-1}^{\prime \prime}}{2 \pi(q)}
$$

which has the solution

$$
\begin{aligned}
\phi_{n}(q) & =-[\pi(q)]^{-1 / 2} \int_{q_{0}}^{q} \frac{\phi_{n-1}^{\prime \prime}\left(q^{\prime}\right)}{2\left[\pi\left(q^{\prime}\right)\right]^{1 / 2}} d q^{\prime} \\
& =\left(\frac{1}{2 \pi} \frac{\partial \Theta}{\partial q}\right]^{1 / 2} A_{n}(q) .
\end{aligned}
$$

This allows us to find $\phi_{n}$ recursively to all orders. We thus obtain

$$
\begin{aligned}
\psi(q, I ; B)= & \exp \left(\frac{1}{\epsilon} S(q, I ; B)\right]\left(\frac{1}{2 \pi} \frac{\partial \Theta}{\partial q}\right)^{1 / 2} \\
& \times\left(1+\sum_{K=1}^{\infty} A_{K} \epsilon^{K}\right),
\end{aligned}
$$

where

$$
\begin{aligned}
& A_{K}(q)=-\int_{q_{0}}^{q} d q^{\prime} \frac{\left[A_{K-1}\left(q^{\prime}\right) \pi\left(q^{\prime}\right)^{-1 / 2}\right]^{\prime \prime}}{2\left[\pi\left(q^{\prime}\right)\right]^{1 / 2}}, \\
& A_{0}=1
\end{aligned}
$$

and the action satisfies the quantization condition $I=(n+\mu) \hbar$.

Since (A10) is an asymptotic series which does not converge $^{14}$ we would in actual practice cut off the series at some $K=M$. Realizing this, we retain the infinite series and consider them as formal expressions.

Now we are ready to write down the expansion for the phase one-form:

$$
A_{\text {Berry }}=\frac{\operatorname{Im}\left[\int d q \psi^{*}(q ; B) d_{B} \psi(q ; B)\right]}{\int d q \psi^{*}(q ; B) \psi(q ; B)} .
$$

Using (A10) and keeping only odd powers of $\epsilon$, which are pure imaginary, we get 


$$
\begin{aligned}
A_{\text {Berry }}= & \frac{1}{i}\left\{\int \frac { d \Theta } { 2 \pi } \left[\frac{d_{B} S}{\epsilon}\left[1+\sum_{k=1}^{\infty} 2 A_{2 k} \epsilon^{2 k}+\sum_{\substack{k, l=1 \\
k+l \text { even }}}^{\infty} A_{k} A_{l}(-1)^{l} \epsilon^{k+l}\right]\right.\right. \\
& \left.\left.+\sum_{k=1}^{\infty} \epsilon^{2 k-1} d_{B} A_{2 k-1}+\sum_{\substack{l, k=1 \\
l+k \text { odd }}}^{\infty}(-1)^{l} \epsilon^{k+l} A_{l} d_{B} A_{k}\right]\right\} \\
& \times\left[\int \frac{d \Theta}{2 \pi}\left[1+\sum_{k=1}^{\infty} 2 A_{2 k} \epsilon^{2 k}+\sum_{\substack{k, l=1 \\
k+l \text { even }}}^{\infty} A_{k} A_{l}(-1)^{l} \epsilon^{k+l}\right]\right]^{-1} .
\end{aligned}
$$

The first four terms of this series are

$$
\begin{aligned}
A_{\text {Berry }}= & \left.\left.\frac{1}{\hbar} \int \frac{d \Theta}{2 \pi} d_{B} S+\hbar\left[\int \frac{d \Theta}{2 \pi}\left[\left(-2 A_{2}+A_{1}^{2}\right) d_{B} S-d_{B} A_{1}\right]-\iint \frac{d \Theta}{2 \pi} d_{B} S\right] \iint \frac{d \Theta}{2 \pi}\left(A_{1}^{2}-2 A_{2}\right)\right]\right] \\
+ & +\hbar^{3}\left[\int \frac{d \Theta}{2 \pi}\left[\left(2 A_{4}-2 A_{1} A_{3}+A_{2}^{2}\right) d_{B} S+d_{B} A_{3}+A_{2} d_{B} A_{1}-A_{1} d_{B} A_{2}\right]\right. \\
& +\int \frac{d \Theta}{2 \pi}\left(-2 A_{2}+A_{1}^{2}\right) \int \frac{d \Theta}{2 \pi}\left[d_{B} S\left(2 A_{2}-A_{1}^{2}\right)+d_{B} A_{1}\right] \\
& \left.+\int \frac{d \Theta}{2 \pi}\left[-2 A_{4}+2 A_{1} A_{3}-A_{2}^{2}+\left(2 A_{2}-A_{1}^{2}\right)^{2}\right] \int \frac{d \Theta}{2 \pi} d_{B} S\right]+\cdots
\end{aligned}
$$

In (A12) and (A13) the integrand should be understood as a function of $(\Theta, I ; B)$. Following Berry ${ }^{2}$ we can introduce the single-valued functions $(0 \leq \theta \leq 2 \pi)$

$$
\begin{aligned}
& \mathcal{S}(\Theta, I ; B)=S(q(\Theta, I ; B), I ; B), \\
& \mathfrak{U}_{K}(\Theta, I ; B)=A_{K}(q(\Theta, I ; B), I ; B),
\end{aligned}
$$

and write

$$
\begin{aligned}
d_{B} S(q, I ; B)= & d_{B} \mathcal{S}(\Theta, I ; B)-p d_{B} q(\Theta, I ; B) \\
d_{B} A_{K}(q, I ; B)= & d_{B} \mathfrak{N}_{K}(\Theta, I ; B) \\
& -\frac{\partial A_{K}(q, I ; B)}{\partial q} d_{B} q(\Theta, I ; B) .
\end{aligned}
$$

Alternatively, we could replace $d \Theta / 2 \pi$ by $c d q / \pi(q)$ in these expressions and consider them as sums of integrals over branches of the torus, the integrands being functions of $(q, I ; B)$.

Now we can use (A12) to find out how the various terms in $A_{\text {Berry }}$ change under a Hannay angle gauge transformation, i.e., under a shift in the origin of the angle variable

$$
\Theta(I ; B) \rightarrow \Theta(I ; B)+\Theta_{0}(I ; B) .
$$

In the expression (A4) for $S(q, I ; B)$ the point $q_{0}$ [or better $\left.\left(q_{0}, p_{0}\right)\right]$ is a reference point for which $S\left(q_{0}, I ; B\right)=0$ and $\Theta\left(q_{0}, p_{0} ; B\right)=0$. Changing the origin of the angle variable corresponds to shifting the reference point

$$
\left(q_{0}, p_{0}\right) \rightarrow\left(\bar{q}_{0}, \bar{p}_{0}\right)
$$

so that

$$
\begin{aligned}
S(q, I ; B) & \rightarrow S(q, I ; B)+\int_{\bar{q}_{0}}^{q_{0}} p\left(q^{\prime}, I ; B\right) d q^{\prime} \\
& =S(q, I ; B)+f(I ; B),
\end{aligned}
$$

where

$$
\frac{\partial f(I ; B)}{\partial I}=\Theta_{0}(I ; B)
$$

and, therefore,

$$
d_{B} S(q, I ; B) \rightarrow d_{B} S(q, I ; B)+d_{B} f(I ; B) .
$$

Since obviously $f(I ; B)$ is independent of $q$

$$
\frac{\partial S(q, I ; B)}{\partial q} \rightarrow \frac{\partial S(q, I ; B)}{\partial q}
$$

and since all the amplitudes $A_{K}$ depend on $q$ derivatives of $S$, we have

$$
\begin{aligned}
& A_{K}(q, I ; B) \rightarrow A_{K}(q, I ; B), \\
& d_{B} A_{K}(q, I ; B) \rightarrow d_{B} A_{K}(q, I ; B) .
\end{aligned}
$$


Substituting (A17) and (A16) into (A12), we find that, under a Hannay angle gauge transformation,

$$
A_{\text {Berry }} \rightarrow A_{\text {Berry }}+\frac{1}{\hbar} d_{B} f(I ; B)
$$

and only the $O(1 / \hbar)$ term in $A_{\text {Berry }}$ gets transformed. The reader may satisfy himself by looking at (A13) that the higher-order terms in $A_{\text {Berry }}$ remain unaffected by (A14).

It would also be interesting to investigate the dependence on $I$ of the higher-order terms in $A_{\text {Berry }}$. Is it possible to show that if Hannay's angle is zero then Berry's phase is independent of $I$, and hence of $n$, to all orders? We leave this question open.
${ }^{*}$ Present address: Physics Department, Tel-Aviv University, Ramat Aviv, Tel-Aviv, Israel.

${ }^{1}$ M. V. Berry, Proc. R. Soc. London A392, 45 (1984).

2J. H. Hannay, J. Phys. A 18, 221 (1985); M. V. Berry, ibid. 18, 15 (1985).

${ }^{3}$ R. Jackiw, Comments At. Mol. Phys. 21, 71 (1988); Int. J. Mod. Phys. A 3, 285 (1988).

${ }^{4}$ E. Gozzi and W. D. Thacker, in Skyrmions and Anomalies, proceedings of the Workshop, Krakow, Poland, 1987, edited by M. Jezabek and M. Praszalowicz (World Scientific, Singapore, 1987); Phys. Rev. D 35, 2388 (1987); 35, 2398 (1987).

${ }^{5}$ S. Chaturvedi, M. S. Sriram, and V. Srinivasan, J. Phys. A 20, L1071 (1987).

${ }^{6}$ G. Giavarini and E. Onofri, J. Math. Phys. (to be published). ${ }^{7}$ W. Magnus, Commun. Pure Appl. Math. 7, 649 (1954).
${ }^{8}$ J. Anandan and L. Stodolsky, Phys. Rev. D 35, 2597 (1987).

${ }^{9}$ R. Casalbuoni, Nuovo Cimento A 33, 115 (1976); 33, 389 (1976); F. A. Berezin and M. S. Marinov, Ann. Phys. (N.Y.) 104, 336 (1977).

${ }^{10}$ B. G. Wybourne, Classical Groups for Physicists (Wiley, New York, 1974).

${ }^{11}$ E. Inönü and E. P. Wigner, Proc. Natl. Acad. Sci. U.S.A., 39, 510 (1953).

${ }^{12}$ V. P. Maslov and M. V. Fedoriuk, Semiclassical Approximation in Quantum Mechanics (Reidel, Dordrecht, 1981).

${ }^{13}$ M. V. Berry, in Chaotic Behavior in Deterministic Systems, edited by R. H. G. Hellman and R. Stora (North-Holland, Amsterdam, 1983).

${ }^{14}$ N. Fröman and P. W. Fröman, JWKB Approximation Contributions to the Theory (North-Holland, Amsterdam, 1964). 